\title{
Is Myanmar on the right track after declaring leprosy elimination? Trends in new leprosy cases (2004-2018) and reasons for delay in diagnosis
}

\author{
MYOKOKO ZAW ${ }^{\mathrm{a}}$, SRINATH SATYANARAYANA ${ }^{\mathrm{b}}$, \\ KYAW KOKO HTET ${ }^{\mathrm{C}}$, KYUKYU THAN ${ }^{\mathrm{d}}$ \& \\ CHAN TUN AUNG ${ }^{\mathrm{a}}$ \\ ${ }^{a}$ National Leprosy Control Programme, Department of Public Health, \\ Ministry of Health and Sports, Myanmar \\ ${ }^{\mathrm{b}}$ Center for Operational Research, International Union Against Tuberculosis \\ and Lung Disease, Paris, France \\ ${ }^{\mathrm{c}}$ Department of Medical Research, Ministry of Health and Sports, Myanmar \\ ${ }^{\mathrm{d}}$ Burnet Institute, Myanmar
}

Accepted for publication 20 November 2019

\begin{abstract}
Summary
Background: Myanmar declared leprosy elimination in 2003. In 2018, the country reported 2,214 new leprosy cases of whom 263 had Grade-2 disability (G2D). The country aims to reduce new cases with G2D to $<53$ cases by 2023 through early diagnosis and treatment.

Objectives: To describe the trends (actual and projected) in new leprosy cases from 2004 to 2023 and to explore the reasons for delay in diagnosis.

Methods: Mixed-methods study. The quantitative part—ecological study using numbers of new leprosy cases from 2004-2018. For qualitative part, new cases with G2D and health care providers were interviewed.

Results: The annual total new cases, new multibacillary cases, new cases with G2D, new child cases are showing a declining trend. Total new cases and new cases with G2D, between 2004 and 2018 have declined by $40 \%$ and $28 \%$ respectively. If the current trends continue there will be 1,785 (95\% CI: 1546-2024) new cases and $<53$ new cases with G2D in 2023. The interviews of patients and providers yielded the following reasons for delay in diagnosis: incorrect knowledge about leprosy, delay in recognition of symptoms, inappropriate care seeking, and fear of stigma and discrimination. Reduction in health system efforts, lack of local leadership, human resource constraints and decreased funding also contributed to delay in diagnosis.

Conclusions: The declining trends and the possibility of achieving the target for new cases with G2D by 2023 is encouraging. The country must address the challenges mentioned by patients and health care providers as a priority, to accelerate the decline in new cases.
\end{abstract}

Keywords: Leprosy, Myanmar, trends, grade-2 disability, delay

Correspondence to: Myo Ko Ko Zaw, Disease Control Building, Zabu Kyetthayay Road, Zabu Thiri Township, Nay Pyi Taw, Myanmar (Tel.: +95-9-979000996; e-mail: drmkkz@gmail.com) 


\section{Introduction}

Leprosy is a curable, chronic, deforming infectious disease caused by the bacterium, Mycobacterium leprae. ${ }^{1}$ Leprosy has been affecting humans since time immemorial and due to the deformities, individuals and communities endure various forms of medical, social and economic hardships. ${ }^{2,3}$

At the beginning of the 20th century, leprosy was a public health problem in many countries of the world. Due to advancements in the knowledge on prevention, clinical diagnosis and treatment, accompanied by improvements in health systems and socio-economic status, by the end of the 20th century, leprosy was eliminated as a public health problem at the global level (elimination as a public health problem was defined as prevalence of $<1$ case per 10,000 population). ${ }^{1}$ However, according to reports received from 150 countries by the World Health Organization (WHO), the global prevalence of leprosy at the end of 2017 was 192,713 cases ( 0.25 cases per 10,000 population) with leprosy still persisting in certain marginalised and vulnerable low income communities. Among these leprosy cases, the disability due to leprosy is signified by Grade-2 disability (G2D). G2D is defined as the presence of visible damage or disability due to leprosy. Although there are decreasing trends of G2D cases globally from 2.5 cases to 1.6 cases per million population, it still remains above the global target of 1 per million population, so is of public health importance. ${ }^{4}$

Myanmar, a country in south-east Asia, achieved the target for leprosy elimination as a public health problem at the national level in 2003. ${ }^{5}$ In the year 2018, Myanmar reported 2,214 new leprosy cases with 263 (11.9\%) having G2D at the time of diagnosis, indicating delay in treatment seeking, diagnosis and/or treatment initiation. The National Leprosy Control Strategy (NLCS) (2019-2023) has set a national goal of reducing new cases with G2D to $<1$ case per million population (i.e., $<53$ cases per year nationally) to be achieved by the year 2023 . This entails a five/six-fold reduction in the number of new cases with G2D to be achieved within a 5-year time frame. ${ }^{5}$

In addition, it is important to monitor the incidence of two other sub-groups of new leprosy cases - multibacillary (MB) cases and child cases. MB cases have higher bacterial load, are more infectious and more likely to be responsible for leprosy transmission, while new child cases (aged $<15$ years) indicate recent transmission. An increase in child cases highlights the presence of active leprosy cases in the community. ${ }^{6}$

In this context, it was necessary to analyze the trends in the new leprosy cases (with special focus on G2D) to estimate the numbers of new leprosy cases that may occur in the next 5 years and if the current trends continue, whether the country will be able to achieve the national target of $<1$ new case with G2D per million population within the next 5 years. Furthermore, there was a need to understand the distribution of new leprosy cases in various States and Regions of the country and assess the reasons for delay in diagnosis. This additional information will help in assessing whether the existing case finding strategies are adequate (or not) for achieving national goals. Therefore, we undertook an operational research study with the following three objectives: first, to assess the trends in new leprosy cases reported in Myanmar from 2004 to 2018 and use this information to make projections for the years 2019 to 2023; second, to describe the new leprosy case burden in various States and Regions of Myanmar for the year 2018 and third, to explore reasons for delay in diagnosis among new leprosy cases with G2D. 


\section{Methods}

STUDY DESIGN

We used a concurrent mixed methods study design ${ }^{7}$ in which the quantitative and qualitative components were carried out in parallel. The quantitative component was an ecological study using data about new leprosy cases reported from 2004-2018. For the qualitative component, we interviewed new leprosy cases with G2D, and leprosy related health care providers, in States/Regions with high G2D burden.

\section{SETTING}

\section{General setting}

Myanmar is a low- and middle-income country with a GDP per capita of USD 1,299 in $2017 .{ }^{8}$ It is the second largest country (256,112 square miles) in South-east Asia and had a population of 51 million in 2014. It has seven States, seven Regions and one Union Territory, Nay Pyi Taw, which is the administrative capital of the country. The country is administratively sub-divided into districts, townships, wards/villages tracts and villages. Seventy percent of the population lives in rural areas. ${ }^{9}$

\section{Study setting}

For the quantitative part of the study, we used aggregate data on new leprosy cases reported from all parts of the country. For the qualitative part, we conducted the study in the six high leprosy burden regions: Yangon, Mandalay, Sagaing, Magway, Ayeyawady and Bago where a total of 1,878 new leprosy cases were detected in 2017 . This accounted for $82.4 \%$ of total new cases in the country. ${ }^{10}$

\section{The Myanmar National Leprosy Control Programme}

In 1952, the Myanmar National Leprosy Control Programme (NLCP) started with dapsone monotherapy. In 1986, Multidrug Therapy (MDT) was introduced as a pilot project under the NLCP. At that time, the country reported 222,209 leprosy cases with a prevalence rate of 59.3 cases per 10,000 population. In 1988, the MDT programme was expanded to six hyperendemic regions and full nationwide coverage was achieved in 1995. After full nationwide coverage with MDT, the country achieved the leprosy elimination target in 2003 (prevalence of $<1$ case per 10,000 population). ${ }^{10}$

During the post-elimination era, the NLCP mainly focused on (i) health education to increase community awareness, (ii) active case detection (household contact surveys, school health talks and leprosy awareness campaigns), (iii) passive case detection (voluntary reporting), (iv) MDT therapy for all cases, (v) prevention of disability \& rehabilitation for people with disability, (vi) recording \& reporting systems on case finding and treatment outcomes and (vii) leprosy related health systems research. Case finding and treatment with MDT have been integrated into the general health system and are being mainly carried out by Basic Health Staff with technical support of leprosy specialized staff. Health education on leprosy is carried out through various mass media with emphasis on early signs and symptoms, curability, availability of free-of-charge MDT drugs and prevention of disability by early diagnosis and treatment. ${ }^{11}$ The definitions of a case of leprosy, paucibacillary (PB) and multibacillary (MB) cases and disability grades $(0,1$ and 2$)$ follow the standard WHO recommendations ${ }^{12}$ and these definitions are described in Box 1. 
Box 1 Definition of a case of leprosy, multibacillary leprosy, grade-2 disability under the Myanmar National Leprosy Control Programme

Leprosy is diagnosed when at least one the following cardinal signs and manifestations is present: (i) Definite loss of sensation in a pale (hypo-pigmented) or reddish skin patch, (ii) A thickness of enlarged peripheral nerves, with loss of sensation and/or weakness of the muscles supplied by that nerve, and (iii) the presence of acid-fact bacilli in a slit skin smear.

Multibacillary Leprosy (MB) cases: $\mathrm{MB}$ in which it was defined as the presence in any one of the following symptoms: skin patch $>5$, any neuritis/neural involvement (irrespective of skin patch) and positive slit skin smear.

Grade-2 Disability (G2D): The G2D is defined as presence of visible damage or disability. For the eyes, this includes the inability to close the eye fully or obvious redness of the eye, visual impairment or blindness. For the hands and feet, visible damage includes wounds and ulcers as well as deformity due to muscle weakness, such as foot drop or claw hand and loss or partial reabsorption of fingers and toes.

Standardized leprosy control reports containing information on the number of new cases detected disaggregated by age, sex, $\mathrm{PB} / \mathrm{MB}$ and disability grade and the treatment status/outcome of all cases under treatment (diagnosed previously) are submitted on a monthly basis from rural health centers and townships to the district Team Leader (TB/Leprosy). They are compiled, and a consolidated report is sent to the state/region level, and a compiled report from each of the 14 States \& Regions is sent to the central level. The data/reports submitted to the district, regional and central levels are periodically validated/verified during routine supervisory visits and any inconsistencies or inaccuracies are rectified.

\section{Study population}

For the quantitative component, we used yearly aggregated data on the numbers of new leprosy cases reported under the NLCP from 2004 to 2018 and in various states and regions for the year 2018. The data was obtained from reports compiled at the central level.

For the qualitative component, to understand the patients' perspective on delays in leprosy diagnosis, we conducted in-depth interviews with 24 new cases (aged $>18$ years) with G2D who started leprosy treatment between 1st January to 31st December 2018, from the six high burden Regions. The samples were purposively selected representing residence (urban and rural) and gender (male and female). To understand the health care provider perspectives on the reasons for delay in diagnosis, 18 health staff were interviewed (six Midwives, six Public Health Supervisors II and six Leprosy Inspectors) from the six high burden Regions.

\section{Study variables and source of data}

The variables for the quantitative study were the number of new leprosy cases, MB cases, new child cases and new cases with G2D for each year from 2004-2018, and the same information was compiled for the 14 states/regions for the year 2018.

The qualitative component explored from both the patient and the provider perspectives on reasons for delay, including the patient's socio-economic and cultural influences, knowledge about the disease and health seeking behaviors. The challenges for implementing leprosy control activities (political will, human resources and implementation barriers for leprosy control and treatment activities) were explored during health care provider interviews. 


\section{Data collection}

For the quantitative component, we obtained the aggregated data on all the study variables that were available in an electronic database in Microsoft Excel sheets at the NLCP (central level) at Nay Pyi Taw.

For the qualitative component, in-depth interviews were conducted between September 2018 and March 2019. The data collection team mainly consisted of two interviewers and two note takers who had previous qualitative data collection experiences. The Principal Investigator from the central leprosy team made appointments by telephone with Midwives, Public Health Supervisors II and Leprosy Inspectors in the six Regions, prior to data collection. Once the date and time was confirmed, the interviews were conducted in a place where the respondent felt most convenient.

For the patient interviews, the principal investigator requested the Regional leprosy teams to identify four patients from their respective regions purposively and requested for their convenient date and time for the interviews. All interviews were done after obtaining written informed consent.

The interviews were audio recorded along with note taking. All interviews were conducted in the Myanmar language. Separate interview guides were used for patients and health care providers. The two interview guides are given in the Appendix A. Neither interviewer had any prior relationship with the study participants.

\section{Data analysis}

For the quantitative component, data analysis was conducted using Microsoft Office Excel version 2010 and STATA (version 12.1 STATA Corp., College Station, TX, USA). We assessed the trends in the yearly number of new leprosy cases, MB cases, new child cases and new cases with G2D over a period of 15 years from 2004-2018. In addition, we also assessed the recent trends (from 2014-2018) in the number of new leprosy cases with G2D per million population. We assessed whether the trends followed a linear or a curvilinear pattern (exponential, logarithmic, polynomial or power) and obtained its regression equation/model. The regression model with highest $R^{2}$ value $(>0.70)$ was chosen as the best fit model and used for projecting the number of new cases for the period 2019 to 2023. The State and Region-wise distribution of leprosy cases for the year 2018 was described in numbers, proportion and rates (cases per million population).

For the qualitative component, interview transcripts were prepared in the Myanmar language based on the audio-recording of interviews. Manual descriptive content analysis was done by three study investigators in order to reduce subjectivity and interpretive credibility and the themes identified. The quotes corresponding to the themes are presented to support the study findings.

\section{Ethics approval}

Ethical clearance for this study was obtained from Ethics Review Committee of Department of Medical Research, Ministry of Health and Sports, Myanmar (Ethics/DMR/2018/171) and The Ethics Advisory Group, The Union, Paris, France (49/18). For the quantitative component of the study, which involves the review of secondary survey records, a waiver for informed consent was sought from the ethics committees. For the qualitative component, written informed consent was obtained from each participant and confidentiality and privacy were maintained throughout the study. 




Legend

Blue solid line $\rightarrow$ Total new leprosy cases;

Red solid line $\rightarrow$ Total MB cases:

Blue dotted line $\rightarrow$ linear trend line for total new leprosy cases;

Footnotes

Red dotted line $\rightarrow$ Curvilinear trend line for $M B$ cases

Regression equation in blue font is for linear trend line for total new leprosy cases

Regression equation in red font is for curvilinear trend line for $M B$ cases

$Y$ in the regression equation represents the number of cases; $x$ indicates the year ( $x=1$ for 2004; $x=2$ for 2005;

$R^{2}=$ goodness of fit measure for the regression equation.

Figure 1. Trends in new leprosy cases and MB cases notified in Myanmar from 2004 to 2018 and projected for 2019 to 2023 .

\section{Results}

QUANTITATIVE

\section{Trends in total new leprosy cases}

The trends in the total numbers of new leprosy cases and MB cases reported in Myanmar from 2004 to 2018 are shown in Figure 1. The annual total number of new leprosy cases has declined by about $40 \%$ from 3,756 cases in 2004 to 2,214 cases by 2018 . The total number of new leprosy cases shows a linear declining trend at the rate of 106 cases per year during this 15 -year period. If the long term 15 year-trend continues, by 2023 there will be 1,785 (95\% CI: 1546-2024) new leprosy cases in the country.

\section{Trends in total new multibacillary leprosy cases}

The annual number of new MB cases has declined by about 21\% from 2,241 cases in 2004 to 1,767 cases in 2018. The long term 15-year trend shows a curvilinear declining trend pattern wherein the rate of decline was low during the initial years from 2004 to 2012, but the rate of decline has increased in the recent years. If this long-term trend continues, there will be about 1,182 (95\% CI: 1010-1374) new MB cases in the year 2023.

\section{Trends in total new leprosy cases with Grade-2 disability (G2D)}

The trends in the numbers of new cases with G2D and new child cases reported in the country from 2004 to 2018 are shown in Figure 2. The annual total number of new cases with G2D has declined by about $28 \%$ from 362 cases in 2004 to 263 cases by 2018 . The decline shows a curvilinear pattern wherein there was an increasing trend in the number of new cases with G2D from 2004 to 2012 and thereafter from 2012 onwards it shows a rapid declining trend. 

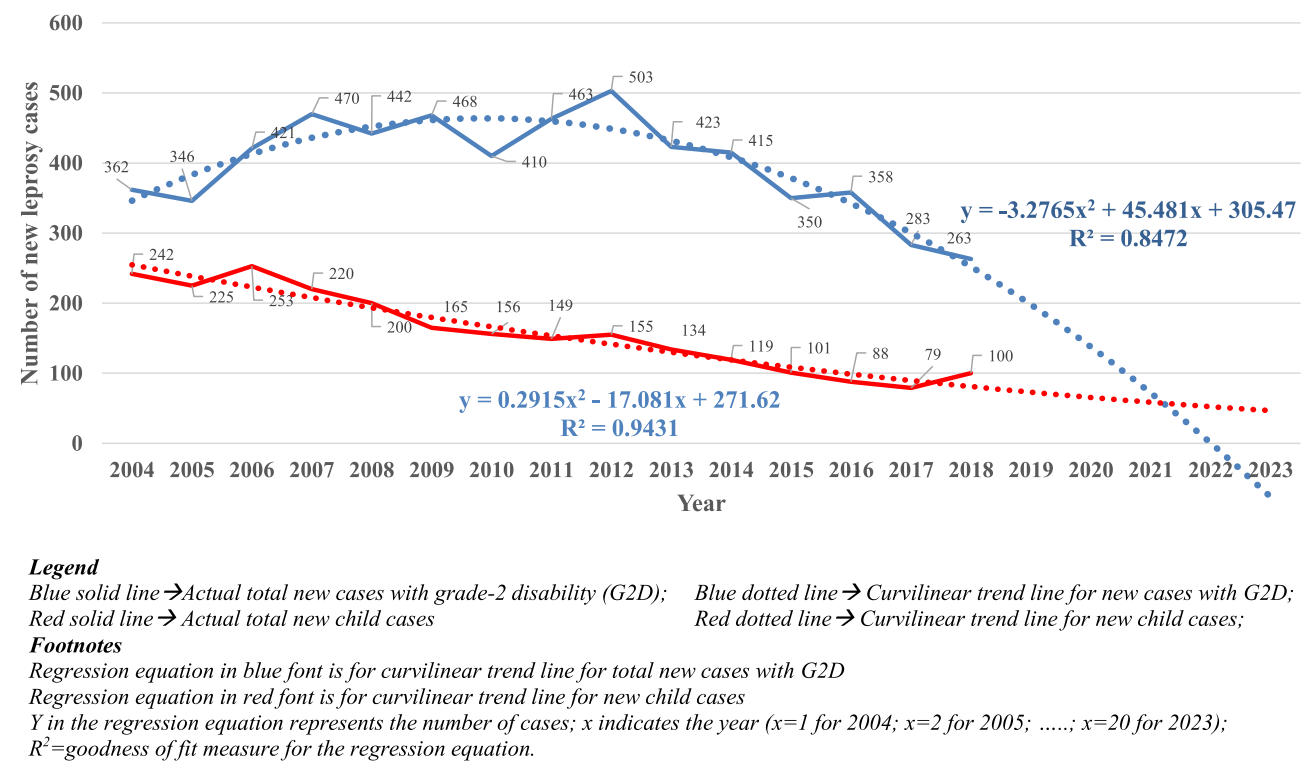

Figure 2. Tends in the numbers of new cases with G2D and new child cases notified in Myanmar from 2004 to 2018 and projected for 2019 to 2023.

If the long-term trend continues, then the country is likely to have zero new cases with G2D by 2022. Since achieving $<1$ new case with G2D per million population is one of the key indicators of the NLCS (2019-2023), we assessed whether the short-term (last 5 years) trend in this indicator also confirms zero cases by 2022 (Figure 3). The short-term trends, however, appears to follow a linear or exponential curvilinear declining trend pattern and these shortterm trends show that 'zero cases' is unlikely to be achieved by 2022 and there may be still 1.5-3 new cases with G2D per million population by 2022. The short-term trends show that $<1$ new case with G2D per million populations is likely to be achieved by 2023 if the declining trend follows a linear declining pattern and beyond 2023 if it follows an exponential curvilinear declining pattern.

\section{Trends in total new child cases (aged $<15$ years)}

The number of new child cases has declined by 60\% from 2004-2018 (Figure 2). The decline shows a curvilinear trend with the rate of decline slowing from 2015 onwards. If this long term 15-year trend continues, there will be about 37 (95\% CI: 8-66) new child cases in 2023.

\section{Distribution of leprosy cases in various States and Regions}

We present the State and Regional-wise distribution of the numbers of new leprosy cases, new MB cases, new child cases, new cases with G2D, rate of new cases with G2D (per million population) and new child cases with G2D in 2018 (Table 1). This table shows that the leprosy burden is highest in Mandalay Region (which includes Nay Pyi Taw union territory) followed by Yangon, Sagaing, Bago, Magway, Ayeyawady and Shan. In three States (Rakhine, Kachin and Chin), there were no new child cases and no new cases with G2D. 


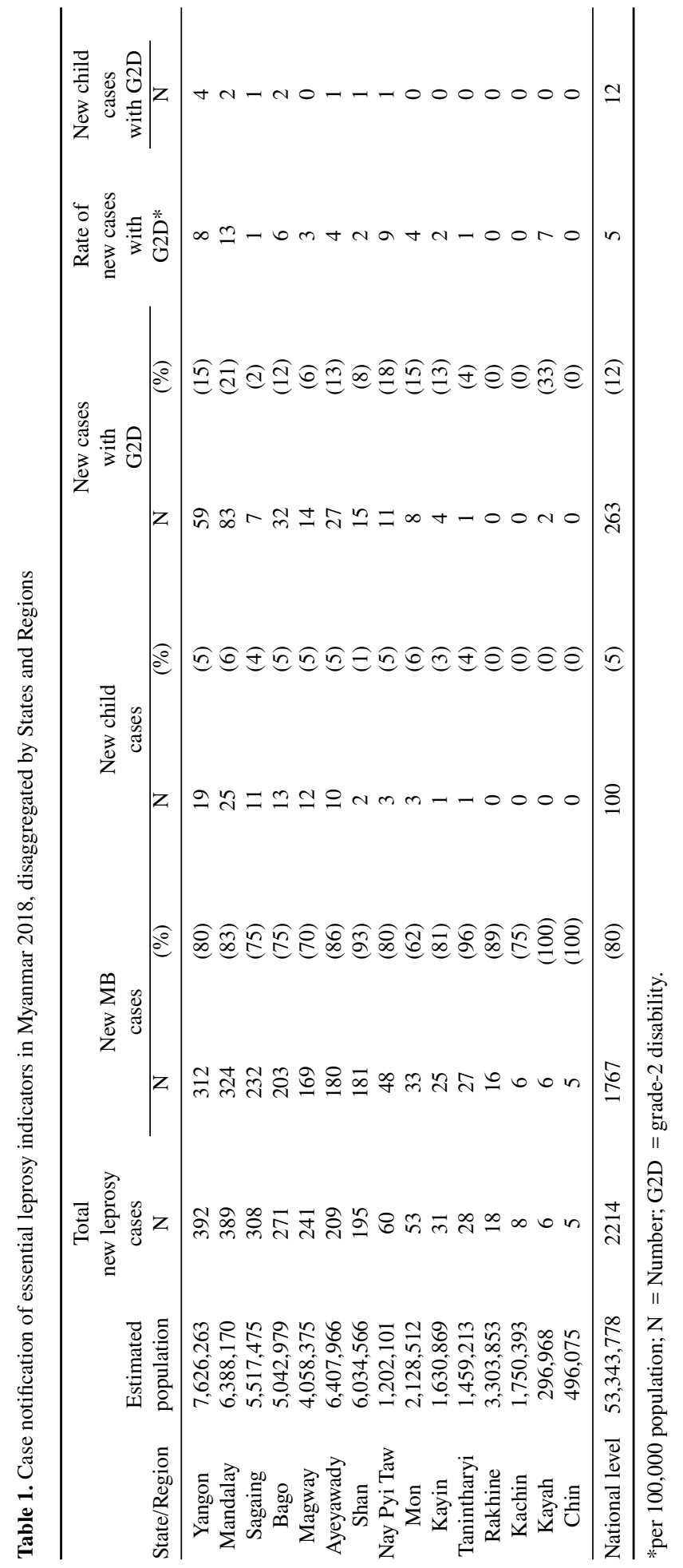




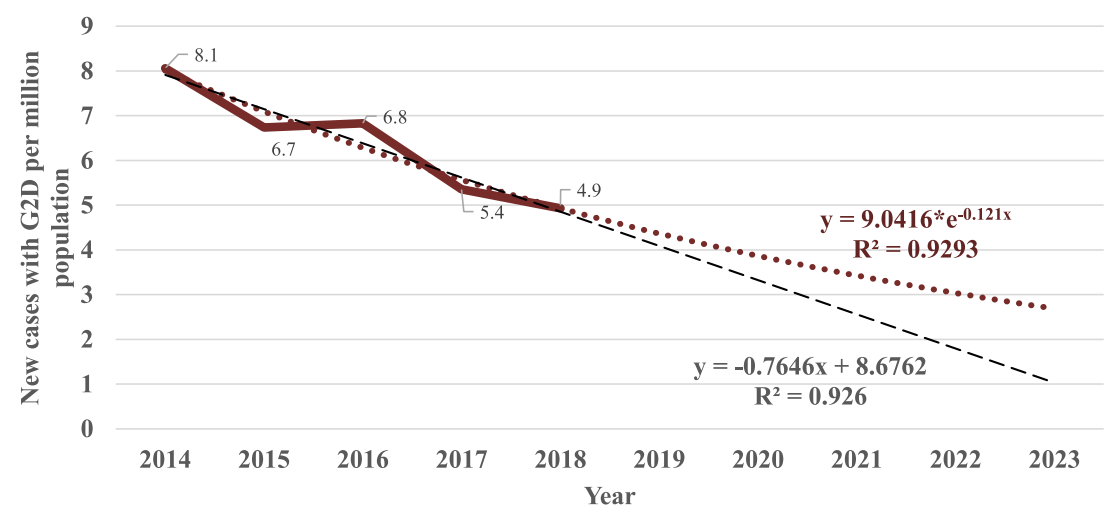

Legend

Solid line indicates the rate of actual new cases with grade-2 disability (G2D); dotted line indicates the curvilinear trend line; dash line indicates the linear trend line;

Footnotes

$Y$ in the regression equation represents the number of cases; $x$ indicates the year $(x=1$ for $2004 ; x=2$ for $2005 ; \ldots . . ; x=20$ for 2023$)$; $R^{2}=$ goodness of fit measure for the regression equation.

Regression equation in red font is for curvilinear trend line for rate of new cases with G2D;

Regression equation in black font is for curvilinear trend line for rate of new cases with G2D;

Figure 3. Trends in rate of new cases with G2D (per million population) notified in Myanmar from 2014 to 2018 and projected for 2019 to 2023.

Table 2. Characteristics of new cases with G2D selected for in-depth interviews for assessing the reasons for delay in diagnosis

\begin{tabular}{|c|c|c|}
\hline Characteristic & $\mathrm{N}$ & $(\%)$ \\
\hline Total & 24 & (100) \\
\hline Median Age in years (Range) & $29(19-53)$ & \\
\hline \multicolumn{3}{|l|}{ Gender } \\
\hline Males & 19 & (79) \\
\hline Females & 5 & (21) \\
\hline \multicolumn{3}{|l|}{ Education } \\
\hline No Schooling & 3 & (13) \\
\hline $1-5$ years & 13 & (54) \\
\hline $5-10$ years & 8 & (33) \\
\hline \multicolumn{3}{|l|}{ Marital status } \\
\hline Not married & 13 & (54) \\
\hline Married & 11 & (46) \\
\hline \multicolumn{3}{|l|}{ Family history of leprosy } \\
\hline Yes & 6 & $(25)$ \\
\hline No & 18 & (75) \\
\hline $\begin{array}{c}\text { Median duration of disease in } \\
\text { years, (Range) }\end{array}$ & $2.5(1-11)$ & \\
\hline
\end{tabular}

\section{QUALITATIVE}

We conducted in-depth interviews with 24 new cases with G2D enrolled for treatment in 2018 to understand the reasons for delay in diagnosis. The characteristics of these patients are given in Table 2. The reasons for delay from the patient perspective pertained to the following themes 
(a) Knowledge about the disease

Many of the patients in the study had incorrect knowledge about the disease. They did not think that their symptoms were serious and related to leprosy. Some of the patients had wrongly believed that someone in the family must have it to contract the disease.

"In 2018 March, I could not open the water bottle. The disease became worse within six month and I could not turn the key to start my car engine. Up to then, I never thought about leprosy because I have no family history." (49 years old, male patient)

"My parents worked at the factory and did not have any knowledge about the disease. My father helped me to treat it using home remedies. Later, one finger dropped off and then two fingers and toes, too. With no remedies left, my life became dead." (22 years old, female patient)

(b) Disease onset related characteristics

Some of the patients did not realize the onset of symptoms and its progression which led them to late diagnosis and delay and seeking care.

"I had a few small white patches on my face. I also suffered numbness of my left hand and that was all. Nothing happened to me ... so it took me one year after the appearance of symptoms to realize that I had this disease [Leprosy]." (30 years old, male patient)

(c) Seeking care

Many patients in the study did not seek appropriate care for their signs and symptoms due to lack of knowledge about disease and its progression. In some cases even though they sought care, they were misdiagnosed and given wrong treatment by health care providers which delayed their diagnosis.

"At first, I suffered weakness of my hand. My parents took me to a traditional healer and I was treated as a stroke patient by using body massage, hot fermentation and puncturing for three months." (18 years old, male patient)

"Something was wrong with my hand...it felt numb and weak. I took traditional medicine for months but it did not improve. After that, I went to a government hospital for a month as I thought that it was due to nerve problems. I also went to private clinics but no one told me that it was due to leprosy" (49 years old, male patient)

Another reason for delay in seeking care was due to economic difficulties as some of the patients were 'hand-to-mouth' workers. A 27 year old working male said that

"I was working at a tea shop day and night and there was no one who could replace me. I did not have any extra money to go to the clinic either"

(d) Fear of stigma and discrimination

Patients also mentioned their fear of knowing the disease as the family and community might discriminate and stigmatize them. A 21 year old boy told his story in sadness:

"I was 19 years old when my hands started curling. I was shy and afraid that my family will be looked down because of me. Since then I stayed inside the house for nearly three years...." (21 years old, male patient)

Eighteen health care personnel were interviewed to find out their perspectives about the challenges for implementing leprosy control activities aimed at early diagnosis and treatment. The main challenges could be categorized broadly into community and health system barriers.

(a) Community barriers

Health care providers mentioned the difficulties in organizing leprosy awareness campaigns at the community level and that the interest and awareness of leprosy has reduced after elimination. It is challenging to give messages about leprosy and still there is considerable stigma about leprosy disease. 
"It's really hard to gather people and get their interest for leprosy education ... so we have to use many tactics to gather them such as giving medical treatment and injections" (Leprosy staff 3)

"Stigma is still there... people are still afraid to find out about the disease... a girl was just diagnosed and she cried heavily saying that she has no family history...it was hard to convince not only the patient but also to make them understand" (leprosy staff 4)

Another area of concern for the health care providers is weak involvement of the local authority. The health staff in the interview stated that it was challenging to get local authorities in any community events organized to improve the awareness levels.

"Local authority is very important....some don't give any help....they are not interested at all” (Basic Health Staff 1)

"Also getting help from the authority is not an easy job...we have to find the right person (a religious leader) and sometime an influential person” (Leprosy staff 3)

(b) Health system barriers

Lack of local leadership

Many health staff mentioned the lack of local leadership, interest and commitment towards leprosy as a major challenge after elimination.

"The Central Unit want us to report cases regularly...but there is no team leader and I am also a new midwife in this township... but in the leprosy team there is no leader and no follower either... not like AIDS, TB and Malaria programs where they have a strong team and a lot of money... Leprosy used to be under a big "Golden Umbrella" once like them but now there is no one at the township level" (Basic Health Staff 3)

Human resource barrier

Health care providers mentioned that limited specialized leprosy staff at the field level was a constraint in conducting leprosy related activities. Health staff mentioned that non availability of staff to do exclusive leprosy related work is a major barrier for early diagnosis

"Because we don't have an assigned Leprosy staff in the townships anymore, we have difficulties in getting monthly reports... We need the help of the Township Medical Officer because he has the authority ... without his help, community and other BHS are not willing... we really need TMOs voice and guidance" (Leprosy staff 3)

Referral centers for Leprosy treatment

Another barrier was the limited number of referral centers for providing appropriate leprosy care. The health staff said that it was difficult to provide timely and appropriate care for the patients as there are only limited referral centers.

"There are only three main referral centers for leprosy; Yangon, Mandalay and Nay Pyi Taw. We don't have a specific team any more ...[for diagnosis and providing care] ....: (Leprosy staff 1)

Travelling and logistic barriers

Difficulties in travelling to do community-based leprosy field activities and monitoring was identified as one of the barriers by health staff in the interviews. The health staff mentioned that due to inadequate necessities for travel, they were unable to carry out field activities

"We face difficulties going to the field... the transportation cost are high and we cannot always pay out of pocket for supervision... so we just cut most of the trips....” (Basic Health Staff 5)

"...so we have to go along with other activities [for doing leprosy work] for example like school health...” (Leprosy staff 1) 


\section{Discussion}

This is the first study from Myanmar assessing the trends in the various forms of new leprosy cases reported in the country during the post-elimination era and also assessing the reasons for delay in diagnosis of leprosy from the patient and health systems perspective.

The major strength of this study is the use of a mixed-methods study design which not only helped us to describe the trends in various forms of new leprosy cases but also explore the reasons for delay in diagnosis that are most commonly responsible for new cases with G2D.

There are two major limitations of this study: First, the declining trends may not reflect reality if there are missing data or underreporting of leprosy cases. This can happen when there is a decline in the focus on leprosy within the generalized health system. ${ }^{13}$ The NLCP is cognizant of this issue and has been making several efforts to address this through strengthening the Register, Record and Report (3Rs) system, and therefore the instances of under reporting of cases is likely to be very minimal. Second, we only interviewed a convenient sample of new leprosy patients with G2D and there could be selection bias. Furthermore, we did not interview patients without G2D to see if there were any facilitators for early diagnosis. Therefore, we are unable to comment whether the reasons for delay quoted by persons with G2D were unique to them or ubiquitous to all leprosy cases in the community.

Despite these two major limitations, the study highlights five important findings that are of public health relevance.

First, all forms of new leprosy cases show a declining trend, and this is highly encouraging. This is in line with the decline in leprosy cases observed at the global level. ${ }^{4}$ However, it was expected that following the achievement of elimination targets, the disease would die out naturally over a period of $10-15$ years due to reduction in disease transmission. ${ }^{14}$ But this has not been the case in Myanmar and the trend analysis indicates that there will still be about 1,785 new cases of leprosy in the year 2023 (20 years after declaring Leprosy elimination in the country). Of particular concern is the high proportion of new cases with multibacillary disease and the occurrence of leprosy cases among children (aged $<15$ years). The former type of case indicates the potential for disease transmission and the latter type indicates the evidence of recent/on-going transmission in the community. ${ }^{15}$ Therefore, despite the achievement of the elimination target $(<1$ case per 10,000 population), the country has not yet reached zero transmission levels. The NLCP needs to examine this issue and devise mechanisms to reduce the transmission levels in the community. The country has participated in the single dose rifampicin, post exposure prophylaxis pilot project. This project demonstrated that it is operationally feasible to give post exposure prophylaxis and is acceptable to the community and this is being scaled up across the country. This will improve the contact tracking and potentially reduce the incidence and transmission of leprosy in due course.

Second, leprosy causes human suffering due to the disabilities, especially G2D. Therefore, the country has set a target of achieving $<1$ new case with G2D per million population to be achieved by 2023 . The country may or may not achieve this target depending on whether the reduction in the number of new cases with G2D follows the long term 15-year trends or the short-term 5-year trends. Early diagnosis and treatment are essential to prevent G2D and this also has the potential to reduce transmission levels in the community. ${ }^{15}$ In our findings, inappropriate knowledge about leprosy and care seeking, social stigmatization, minimal community participation and interest about leprosy and lack of leprosy specialized staff, emerged as the main reasons for delay in diagnosis of leprosy, leading to G2D. Therefore, in order to reduce the burden of new cases with G2D these issues have to be addressed by the NLCP. 
Third, the burden of leprosy is concentrated in six Regions and one State of the country. There have not been any studies in the country describing the profile of leprosy cases occurring in the community in the recent past. Therefore, very little is known about who is getting affected by leprosy, their geographic distribution, whether there is any geographic clustering and why they are getting affected by the disease. Investigating these issues is an area for future operational research. This will help in better targeting of the NLCP services to the persons/communities more in need of these services.

Fourth, several patient level, community level and health system level issues were quoted by patients and health care providers as responsible for late detection of leprosy cases. Of them, the notable ones are inappropriate levels of knowledge about leprosy disease, fear of stigma and discrimination in the community, and reduced focus on leprosy by the health care workers of the general health system. The NLCP will have to find out if strategies outlined within the NLCS (2019-2023) are adequate or if there are better ways of reaching the population with leprosy messages. This is an area for future operational research.

Lastly, the study findings call upon the various global stakeholders involved and responsible for leprosy elimination/eradication to continue to maintain their vigilance on the incidence of leprosy cases across the world. ${ }^{15}$ Leprosy is still an unfinished business and is responsible for unnecessary suffering among a significant number of people. We feel that our study will stimulate other countries to assess and report on the trends in leprosy cases from their own settings. This will help in better understanding of the current leprosy scenario in various parts of the world.

\section{Conclusion}

The numbers of new leprosy cases in Myanmar is showing a declining trend but there will still be about 1,785 new cases in the year 2023 if the current trends continue. The country will be able to achieve the target of $<1$ new case with G2D by 2023. Delays in diagnosis among new cases with G2D are due to poor/incorrect knowledge about leprosy, and inadequate health system efforts to diagnose the cases early. The NLCP needs to address these two issues as a priority.

\section{Acknowledgements}

This research was conducted through the Structured Operational Research and Training Initiative (SORT IT), a global partnership led by the Special Programme for Research and Training in Tropical Diseases at the World Health Organization (WHO/TDR). The model is based on a course developed jointly by the International Union Against Tuberculosis and Lung Disease (The Union) and Medecins Sans Frontieres (MSF/Doctors Without Borders). The specific SORT IT programme which resulted in this publication was jointly organised and implemented by The Centre for Operational Research, The Union, Paris, France; Department of Medical Research, Ministry of Health and Sports, Yangon; Department of Public Health, Ministry of Health and Sports, Nay Pyi Taw; The Union Country Office, Mandalay, Myanmar; The Union South-East Asia Office, New Delhi, India and London School of Hygiene and Tropical Medicine, London, UK.

\section{Funding}

The training programme, within which this paper was developed, was funded by the Department for International Development (DFID), London, UK. The implementation of the research 
was funded by Myanmar Ministry of Health and Sports Research Grant (2018-2019). The funders had no role in study design, data collection and analysis, decision to publish or preparation of the manuscript.

\section{Conflict of interest}

The authors have no conflict of interest to declare.

\section{Author contributions}

- Conceived the study: MKKZ CTA

- Developed the protocol: MKKZ, SS, KKKH, KKT, CTA

- Data collection: MKKZ, KKKH

- Data analysis and interpretation MKKZ, SS, KKKH, KKT

- Manuscript drafting: MKKZ, SS, KKKH, KKT

- Critical review and approval: All authors

\section{Appendix A: Qualitative: Interview guide with patients}

Patient's characteristics: Age, sex, education, marital status, any family member with the leprosy disease, date of first symptom appear and date of start of treatment.

Theme (1): Recognition of the disease

- How did you notice your disease characteristics?

- For how long have you noticed it?

- Did you have a sense of getting leprosy? (Self-perceived risk of contracting leprosy and perceived susceptibility of getting leprosy by family members)

\section{Theme (2): Seeking care}

- Did you discuss your signs and symptoms with anyone? (family members, relatives or neighbors or friends?)

- If not, why? If, yes, who?

- Who suggested that you should seek care? (influencing person to do care and social support to seek health care)

- Where did you seek treatment when you first noticed the symptoms/skin lesions?

- Did you use any home remedies to treat it? If yes, who suggested it, why did you accept to use it?

- Did you visit any health care providers? Did you seek care from any others than health care providers? (private or public)

- If yes, who suggested it and the reasons for seeking care.

\section{Theme (3): Reasons for delay in seeking care}

- How long after you first observed the symptoms did you seek care?

- If long, what are the reasons for the long delay in seeking care?

- Explore reasons for delay in seeking care or diagnosis, whether it was due to lack of awareness, decision delay, financial barriers, geographical barriers, social stigmatization and disclosure related issues etc., 


\section{Theme (4): Family contact}

- Have any members of the family had the disease previously? (immediate or relatives)

- If yes, did they take complete treatment or not?

- Do you think that it is related with a family contact?

\section{Theme (5): Outcome of the disease}

- Where do you think you got the disease from?

- What is your opinion about the disease, early diagnosis and its treatment?

- Belief about the positive outcomes of seeking care (early diagnosis and treatment of leprosy, reduce the consequences of leprosy and disease transmission to family and community)

- Belief about the negative outcomes of seeking care (perception on social stigma, shame and feeling about disclosure)

\section{Appendix B: Qualitative: Interview guide with health care providers}

Providers' characteristics: Age, sex, education, designation, current position service and total service years

Theme (1): Role and responsibility for leprosy control activities

- Please tell me your role and responsibility for leprosy control activities

- Raising community awareness, new case findings, reducing Grade-2 disability cases in the community

\section{Theme (2): Activities performed for finding leprosy cases at the community}

- Who is involved?

- How do you organize it?

- What activities are performed? How frequently?

\section{Theme (3): Community level barriers and constraints for leprosy control activities}

- What are the barriers and constraints at the community level to do leprosy control activities? (Manpower, Financial, Mechanical, Administrative procedures, community and local authority involvement and so on)

\section{Theme (4): Programme level barriers and constraints for leprosy control activities}

- What are the barriers and constraints at programmatic level for leprosy control activities? (Policy trends, Supervision, Monitoring, Evaluation)

\section{Theme (5): Suggestion to get early new leprosy case diagnosis and to prevent disability}

- Can you tell me about possible ways to detect leprosy cases early and get treatment?

\section{References}

1 Lockwood DNJ, Suneetha S. Leprosy: too complex a disease for a simple elimination paradigm. Bull World Health Organ, 2005; 83(3): 230-235, [Internet]. [cited 2019 Oct 22]; Available from: http:// www.ncbi.nlm.nih.gov/pubmed/15798849.

2 World Health Organization. The final push strategy to eliminate leprosy as a public health problem: questions and answers. WHO 2003. 
3 Walker SL, Lockwood DNJ. The clinical and immunological features of leprosy. Br Med Bull, 2006; 77-78(1): 103-121, [Internet]. [cited 2019 Oct 22]; Available from: https://academic.oup.com/bmb/articlelookup/doi/10.1093/bmb/ld1010.

4 World Health Organisation. Global leprosy update, 2016: accelerating reduction of disease burden. In: Weekly epidemiological record. vol. 92, 2017; pp. 501-519.

5 Ministry of Health and Sports. National Leprosy Strategic Plan (2019-2023), Myanmar. 2018.

6 Fine PE. Reflections on the elimination of leprosy. Int J Lepr Other Mycobact Dis, 1992; 60(1): 71-80, [Internet]. [cited 2019 Oct 22]; Available from: http://www.ncbi.nlm.nih.gov/pubmed/1602195.

7 Doyle L, Brady A-M, Byrne G. An overview of mixed methods research - revisited. J Res Nurs, 2016; 21(8): 623-635.

8 World Bank Country and Lending Groups - World Bank Data Help Desk [Internet]. [cited 2019 Oct 22]. Available from: https://datahelpdesk.worldbank.org/knowledgebase/articles/906519.

9 Department of Population. Census Atlas Myanmar. 2014.

10 Ministry of Health and Sports. National Leprosy Control Programme Annual report, Myanmar. 2017.

11 Ministry of Health and Sports. Leprosy Control Guideline for Basic Health Staff. Myanmar: National Leprosy Control Program.

12 Ministry of Health and Sports. National Guideline for Myanmar Leprosy Control Programme, Myanmar.

13 Mallick SN. Integration of Leprosy Control With Primary Health Care, Myanmar. Lepr Rev, 2015; 72(1): 148153.

14 Scollard DM, Adams LB, Gillis TP, Krahenbuhl JL, Truman RW, Williams DL. The continuing challenges of leprosy. Clin Microbiol Rev, 2006; 19(2): 338-381, [Internet]. Available from: http://www.ncbi.nlm.nih.gov/ pubmed/16614253.

15 Chaptini C, Marshman G. Leprosy: a review on elimination, reducing the disease burden, and future research. Lepr Rev, 2015; 86(4): 307-315, [Internet]. Available from: http://www.ncbi.nlm.nih.gov/pubmed/26964426. 